\title{
Implementation of Structural Assemblies by Simultaneous Projection and Density-Based Topology Optimization
}

\author{
Nouman Saeed $\left(D,{ }^{1}\right.$ Kai Long $(D),{ }^{1}$ Jamshed Ahmed Ansari $(i),{ }^{2}$ Nasif Raza Jaffri $\left(i D,{ }^{3}\right.$ \\ and Usama Abrar iD ${ }^{3}$ \\ ${ }^{1}$ State Key Laboratory for Alternate Electrical Power System with Renewable Energy Sources, \\ North China Electric Power University, Beijing 102206, China \\ ${ }^{2}$ Deparment of Electrical Engineering, Sukkur IBA University, Sukkur 65200, Pakistan \\ ${ }^{3}$ School of Control and Computer Engineering, North China Electric Power University, Beijing 102206, China
}

Correspondence should be addressed to Kai Long; longkai1978@163.com

Received 18 June 2021; Revised 27 July 2021; Accepted 18 August 2021; Published 29 September 2021

Academic Editor: Riaz Ahmad

Copyright ( $\odot 2021$ Nouman Saeed et al. This is an open access article distributed under the Creative Commons Attribution License, which permits unrestricted use, distribution, and reproduction in any medium, provided the original work is properly cited.

\begin{abstract}
This article proposed a methodology that combines two well-known projections and modified density-based optimized techniques in one formulation methodology. This methodology contains an effective explicit geometric entity identified by shape variables that provide easy control in desired particular regions; implicit density-based topological optimization entities utilized topological variables that offer critical design elsewhere. Our main attractive key point of this combined formulation approach is structural assemblies. Structure always manufactures in many patches and joins them by utilizing the structural assemblies such as welding and riveting. It is not easy to execute the structural patches at the required region without acknowledging their dimensions. This proposed approach demonstrates the competence to impose the restrictions related to shape and topological variables of interfaces among the specific patches. Numerous standard numerical examples make sure the validation of the introduced methodology. It remarked that the optimal design could minimize compliance and the minimum number of iterations through numerically performed, concerning computational cost minimized without any kind loss of accuracy of the final structure.
\end{abstract}

\section{Introduction}

Topology optimization is well-known computational structural methodology which we used for spawning different fundamental structural design. This methodology has acquired significant evolution in various engineering fields and a wide range of industrial areas. During the previous three decades, the inaugural study of Bendsøe and Kikuchi [1] proposes various numerical techniques and mathematical expressions. Topology optimization is more valuable as parallel to the size and shape optimization. It enables optimized designs of complex geometry that we are incapable of solving and shape optimization. Topology optimization increases with every passing day in industrial engineering, automobile, and aerospace industries sides to achieving minimum weight and maximum stiffness purpose. Despite remarkable advances in computer accomplishments, large-scale topology optimization still faces difficulty due to massive computational costs. In topology optimization, many researchers have put their efforts into decreasing the computational expense regarding their point of view. Nguyen et al. [2] advised the multiresolution topology optimization (MOTP) approach to obtain the optimized design at a lower computational cost. Lieu et al. and Filipov et al. $[3,4]$ mixed up this approach in the framework of isogeometric analysis and polygonal elements' techniques to obtaining optimized structure at a lower cost. Wu et al. [5] suggested a new method of exploring optimization and structural analysis for producing bone-like pours' designs. Long et al. [6] indicated that a well-organized and effective quadratic formulation depends on the standard-type isotropic material with penalization technique utilized to solve transient heat conduction problems. Generally, in the solid isotropic material with penalization (SIMP) technique, 
material properties of every element are described explicitly corresponding to relative density. The structured domain discretized into the number of FE; we used these elements to design variables for the optimization structure. Numerous methods are discussed in depth in topology optimization, including formulations and techniques. For example, the level set technique [7], the (SIMP) approach [8], structural and bidirectional structural optimization $[9,10]$, and metaheuristic methods enabled topology optimization to obtain pleasing layouts. Zhang et al. [11] introduced a fair number of holes in the preferred design domain. These holes improve the inhomogeneity of the structure domain which is helpful to obtaining the optimized microstructure results. Keshavarzzadeh et al. [12] suggested Gaussian process-based methods for solving the stress-based topology optimized. Amir and Shakour [13] described a density-based technique for optimizing the structure of prestressed concrete beams.

Wang et al. [14] suggested an approach for gaining maximum stiffness of continuum design within different moveable hole sizes utilizing the moving morphable bars. Long et al. [15] proposed a reciprocal SIMP technique for stress-constrained optimization continuum structure containing the harmonic load. Identifying the solution of novel systems shows that the significant performance of topology optimization is more efficient than traditional techniques. In the numerous manufacturing technologies, some geometrical characteristics of the optimized structure are suffering from few drawbacks, just like the deficiency of direct geometrical control (indeed, some geometric characteristics of the optimized structure can be controlled implicitly). In comparison, implicit level set and density-based constraints proposed appropriate design freedom. According to the literature review, it is difficult to obtain direct control throughout the resulting geometry by utilizing the simple LSM or density-based approach. Implicitly, topology optimization achieves by using different techniques utilizing existing ways. In the following form, the associated problem is summarized by using the implicit method. First, to provide the structural feature sizes, it is difficult to precisely control the structure although it is imperative for manufacturing viewpoint, under the framework of implied topology optimization [16-24]. This is due to the unavailability of explicit geometry information implied in model optimization and embedded techniques to gain full-length scale control. Furthermore, unique methods must be suggested for convenience and scale control $[25,26]$. Additionally, it is challenging to link computer-aided design (CAD) and optimization models for modeling systems under the implied system or framework. In these two settings, structural geometries are presented differently [27]. Second, three-dimensional problems in the suggested topology optimization approach are more significant due to design variables relatively. More design variables are achieved through higher resolutions. Third, the optimization model and analysis model, in implicit approaches, are always coupled strongly. The problems that occur are checkerboard pattern and spurious local vibration. Sometimes, it can lead to simple numerical glitches that may stop the optimization algorithm from approaching purposeful explanations, notably the multiphysics setting, which is considered a topology optimization problem.

Recently, in the topology optimization process, an alternative so-called geometric projection class is dependent on the explicit distribution of parameters of the structure. Norato et al. [28] suggested a density-based geometric projection technique with differentiable mapping from discrete design variables. Meng and Keshtegar [29] proposed the adaptive conjugate single-loop approach to upgrade the efficiency and robustness of reliability-based design optimization RBDO utilizing the single-loop system based on the conjugate gradient. Besides, Zhang et al. [30] improved this technique for 3D structure execution. Furthermore, Watts and Tortorelli [31] developed a geometric projection approach to lattice material from inverse homogenization. White and Stowel suggested a practical approach to apply Fourier representation in combination with density-based topology optimization. Meng et al. [32] proposed an innovative hybrid RBTO approach to sort out multisource ambiguities, material variations, and assembling errors of geometric dimensions and loads. According to the manufacturing point of view, most of the above-discussed methodology indicates that these are an explicit geometric representation. So, in this way, we got easy access among CAD and optimization models for modeling system under the implied system or framework.

In this article, we combined the projection and density-based optimized formulation methodology. The following main key points are an effective explicit geometric entity identified by shape variables. These variables provide easy control in desired selective regions. On the contrary, implicit density-based topological optimization entities used topological variables that play a critical role in other design parts. There are numerous benefits of combining projection, and density-based optimized formulation methodology can be summarized. First, coupling the shape and topological variables is helpful for prestressed concrete optimization (Amir and Shakour) [13]. This combined formulation approach's main attractive key point is structural assemblies, the structure produced in several patches. After that, it joined them by utilizing structural assemblies such as welding and riveting. It is challenging to execute the structural patches at the required region without acknowledging their dimension. This proposed approach demonstrates the competence to impose the restrictions related to shape and topological variables of interfaces among the specific patches.

The order of this article is as follows. In Section 2, the primary setting of geometric and density based according to sigmoid function entities is explained. Furthermore, we briefly describe the combination methodology for specific control and belonging to interface components in Section 3. Section 4 indicates the sensitivity analysis, and Section 5 briefly discusses the capability of the suggested methodology concerning numerical aspects. Nevertheless, the results are stated in Section 6, respectively. 


\section{Methodology}

2.1. Topology Optimization Formulation. To elaborate on the combination of explicit geometric projection and implicit density-based topology optimization methodology in a unique formulation, a practical, precise geometric entity is identified by shape variables to provide easy control in desired selective regions. On the other side, implicit densitybased topological optimization entities are utilized topological variables that offer critical design elsewhere. Here, geometric positions of nodal coordinates of line segments are utilized. The general mathematical formulation for structural topology optimization problem is [33]

$$
\begin{aligned}
\min _{\rho, \mathbf{x}} & : f(\rho, \mathbf{x}) \\
\text { subject to } & : g_{k}(\rho, \mathbf{x}) \leq 0, \quad k=0, \ldots, m \\
& : 0 \leq \rho_{i} \leq 1, \quad i=1, \ldots, N_{\text {ele }} \\
& : x_{\mathrm{lb}} \leq x_{j} \leq x_{\mathrm{ub}}, \quad j=1, \ldots, N_{\text {node }} \\
& : \mathbf{K}(\rho, \mathbf{x}) \mathbf{u}=\mathbf{f},
\end{aligned}
$$

where $f(\rho, x)$ is an objective function, $x$ and $\rho$ are significant design variable vectors concerning nodal coordinates and densities, where $N_{\text {ele }}$ and $N_{\text {node }}$ are the number of FE besides nodes classifying the projection profile, $x_{\mathrm{lb}}$ denotes the lower bound of the geometric coordinate variable of the node, and $x_{\mathrm{ub}}$ corresponds to its upper bound, respectively. $K$ represents the stiffness matrix, although the symbols $u$ and $f$ are so-called displacement and applied loads, respectively.

2.2. Density-Based Projection Parameterization. Obtain an improved depiction of the optimized topological structure by filtering the finite element mesh to achieve a better representation of the optimized topological structure by filtering the finite element mesh. Low-order element discretized the design ached through the distinguished checkerboard pathway. To prevent such problems, we assume density field variables such as $\rho \longrightarrow \tilde{\rho} \longrightarrow \bar{\rho}$; the field of design variable $\rho$ is regularized to get rid of these checkerboard pathway and mesh dependency through the density filter, which is denoted by $\tilde{\rho}_{i}[34]$. The density filter $\tilde{\rho}_{i}$ components calculate average weight of the neighboring values as

$$
\tilde{\rho}_{i}=\frac{\sum_{j \in N_{e, i}} \omega\left(\Delta \mathbf{x}_{i j}\right) \rho_{j}}{\sum_{j \in N_{e, i}} \omega\left(\Delta \mathbf{x}_{i j}\right)} .
$$

Here, the range of design variable $\rho$ enclosed between one and zero helps differentiate between the solid and void spaces. On the other side, $N_{e, i}$ is the neighborhood set of cells lying in the specified filter domain of cell $i . \omega\left(\Delta x_{i j}\right)$ is a linear weighting function that is the distance between the centroids of the cells $i$ and $j$. The mathematically linear weighting function is defined as

$$
\omega\left(\mathbf{x}_{j}\right)=\mathrm{R}-\left|\mathbf{x}_{j}-\mathbf{x}_{i}\right|,
$$

where $R$ represents the specified filter radius. Taking the derivative of $\tilde{\rho}_{i}$ w.r.t. the density variable field $\rho_{j}$, we obtain as follows:

$$
\frac{\partial \tilde{\rho}_{i}}{\partial \rho_{j}}=\frac{\omega\left(\mathbf{x}_{j}\right)}{\sum_{j \in N_{e, i}} \omega\left(\mathbf{x}_{j}\right)}
$$

Equation (2) can be rewritten in the matrix form as follows:

$$
\widetilde{\rho}=D\left(H_{s}\right)^{-1}(H \rho),
$$

where entries $\left(H_{s}\right)$ and $(H)$ are vectors that are $\left[N_{\text {ele }} \times 1\right]$ and $\left[N_{\text {ele }} \times N_{\text {ele }}\right]$. These vectors are described as

$$
\begin{aligned}
H_{i j} & =\omega\left(\Delta \mathbf{x}_{i j}\right), \\
H_{s} & =\sum_{j} H_{i j} .
\end{aligned}
$$

In this paper, at this stage, to project a parameterized smooth projection function proposed in Guest et al. [18, 35-37], the filtered density regarding smooth Heaviside function that needs to have physical characterizations is mathematically calculated as

$$
\bar{\rho}=\left\{\begin{array}{l}
0 \leq \tilde{\rho_{i}} \leq \eta \\
\eta \leq \tilde{\rho_{i}}<1
\end{array}\right.
$$

In equation (7), the parameter $\eta$ values are lying between zero to one. By introducing the gamma $(\gamma)$ parameter, the physical density can be improved as

$$
\bar{\rho}= \begin{cases}\eta\left[e^{-\gamma\left(1-\tilde{\rho_{i} / \eta}\right)}-\left(\frac{1-\tilde{\rho}_{i}}{\eta}\right) e^{-\gamma}\right], & 0 \leq \tilde{\rho}_{i} \leq \eta, \\ (1-\eta)\left[1-e^{-\gamma\left(\tilde{\rho_{i}-\eta}\right) /(1-\eta)}+\frac{\left(\tilde{\rho}_{i}-\eta\right)}{(1-\eta)} e^{-\gamma}\right]+\eta, & \eta \leq \tilde{\rho}_{i}<1 .\end{cases}
$$

Here, the behavior of equation (8) represents that the smooth Heaviside function is continuous. For $\eta=0$, the estimated assessment of the smooth Heaviside function $\bar{\rho}$ is related to a filtered variable that helps to certify the length scale on the solid region. In contrast, similarly on the opposite side, it helps to ensure the length scale on the solid region at $\eta=1$. Here, the concise and innovative smooth Heaviside function expression that rests on sigmoid function $\sigma$ notwithstanding is traditionally illustrated as tanh function. This naive expression provides more straightforward and faster computing despite the defined smooth Heaviside function in the previous equation (8). This alternative smooth Heaviside sigmoid-based function expression is given as

$$
\begin{aligned}
\sigma(x) & =\frac{b}{1+e^{\left(-a \cdot{ }^{*}(x-c)\right)}}+d, \\
\overline{\rho_{i}} & =\frac{\sigma(\gamma \eta)+\sigma\left(\gamma\left(\tilde{\rho}_{i}-\eta\right)\right)}{\sigma(\gamma \eta)+\sigma(\gamma(1-\eta))},
\end{aligned}
$$

where $a, b, C$, and $d$ are parameters. The partial derivative of the sigmoid-based smooth Heaviside projection regarding the design variable is shown respectively:

$$
\frac{\partial \overline{\rho_{i}}}{\partial \widetilde{\rho_{i}}}=\frac{(a \gamma) \cdot{ }^{*} \sigma\left(\gamma\left(\tilde{\rho}_{i}-\eta\right)\right) \cdot{ }^{*}\left(1-\sigma\left(\gamma\left(\tilde{\rho}_{i}-\eta\right)\right) / b\right)-3 d}{\sigma(\gamma \eta)+\sigma(\gamma(1-\eta))}
$$


By utilizing the robust topology optimization technique (RTO) along eroded $\bar{\rho}^{e}$, intermediate $\bar{\rho}^{i}$, and dilated $\bar{\rho}^{d}$ density vectors which explicitly depend on the represented filter density field $\widetilde{\rho}$ although $\widetilde{\rho}$ filter density also explicitly depends upon on design variable $\rho$, respectively, Lazarov et al. utilize the proposed dilated $\bar{\rho}^{d}$ and eroded $\bar{\rho}^{e}$ density vectors in the concerning problem for quantifying the worst case of volume and stiffness. As mentioned above, eroded $\bar{\rho}^{e}$ and dilated $\bar{\rho}^{d}$ density fields are written as follows:

$$
\begin{gathered}
\bar{\rho}_{i}^{e}=\frac{\sigma\left(\gamma \eta_{e}\right)+\sigma\left(\gamma\left(\tilde{\rho}_{i}-\eta_{e}\right)\right)}{\sigma\left(\gamma \eta_{e}\right)+\sigma\left(\gamma\left(1-\eta_{e}\right)\right)}, \\
\bar{\rho}_{i}^{i}=\frac{\sigma\left(\gamma \eta_{i}\right)+\sigma\left(\gamma\left(\tilde{\rho}_{i}-\eta_{i}\right)\right)}{\sigma\left(\gamma \eta_{i}\right)+\sigma\left(\gamma\left(1-\eta_{i}\right)\right)}, \\
\bar{\rho}_{i}^{d}=\frac{\sigma\left(\gamma \eta_{d}\right)+\sigma\left(\gamma\left(\tilde{\rho}_{i}-\eta_{d}\right)\right)}{\sigma\left(\gamma \eta_{d}\right)+\sigma\left(\gamma\left(1-\eta_{d}\right)\right)},
\end{gathered}
$$

where $\eta_{e}=0.6, \eta_{i}=0.5$, and $\eta_{d}=0.4$ are threshold projection parameters of the eroded intermediate and dilated designs and $\gamma$ governs the sharpness of the projected smooth projection. The minimum value of the gamma parameter is 0.1 . In the optimization procedure, the intended dilated field vector $\bar{\rho}^{d}$ is utilized for evaluating the volume constraint whereas and the eroded $\bar{\rho}^{e}$ density field vector is utilized for the calculation of the compliance and structural stiffness, respectively.

The static equilibrium equation is obtained as the displacement field. This field is calculated due to utilizing the linear equation system:

$$
\mathbf{K}\left(\bar{\rho}^{e}, \mathbf{x}\right) \mathbf{u}=\mathbf{f} .
$$

2.3. Material Interpolation Schemes. Material interpolation approaches make sure the acknowledgment of intermediate material throughout the solution procedure. In topology optimization, usually material properties utilized for intermediate densities and material densities may obtain any suitable value between 1 and 0 .

The material penalization mathematically performs to carry out black and white design. According to Young's Modulus $E$ of each element, the eroded density field vector is computed with the help of the modified solid isotropic material penalization (SIMP) interpolation technique [38]. The idea of the scheme is that

$$
E\left(\bar{\rho}_{i}^{e}\right)=E_{\text {min }}+\left(E_{\max }-E_{\min }\right)\left(\bar{\rho}_{i}^{e}\right)^{\mathrm{PE}},
$$

where $E_{\min }$ is a slight stiffness which is assigned to void spaces concerning the stiffness matrix not to be singular, and usually, PE is used as a solid isotropic material penalization factor which should be between 1 and 3, respectively. Here, taking the derivative of equation (13),

$$
\frac{\partial E\left(\bar{\rho}_{i}^{e}\right)}{\partial \bar{\rho}_{i}^{e}}=E_{\min }+\operatorname{PE}\left(E_{\max }-E_{\min }\right)\left(\bar{\rho}_{i}^{e}\right)^{\mathrm{PE}-1} .
$$

In [39], the so-called RAMP (rational approximation of material properties) approach was recommended as an alternative interpolation approach. Let $E\left(\bar{\rho}_{i}^{e}\right)$ denote the mathematical function used in the topology optimization is given as follows:

$$
E\left(\bar{\rho}_{i}^{e}\right)=E_{\min }+\left(E_{\max }-E_{\min }\right)\left(\frac{\bar{\rho}_{i}^{e}}{\left(1+\operatorname{PE}\left(1-\bar{\rho}_{i}^{e}\right)\right)}\right),
$$

where $E_{\min }$ and $\mathrm{PE}$ is penalization factor which should be $\mathrm{PE} \geq 0$ and $E_{\max }$ is an actual value of Young's Modulus for distribution material in topology optimization:

$$
\frac{\partial E\left(\bar{\rho}_{i}^{e}\right)}{\partial \bar{\rho}_{i}^{e}}=E_{\min }+\left(E_{\max }-E_{\min }\right) \frac{(1+\mathrm{PE})}{\left(1+\mathrm{PE}\left(1-\bar{\rho}_{i}^{e}\right)\right)^{2}} \text {. }
$$

We demonstrate the behaviors of the SIMP and RAMP in Figure 1, regarding to values of PE, respectively. For compliance minimization, we utilize a RAMP penalization instead of SIMP penalization. The output of equations (14) and (16) is obtained at given input zero and 1 regarding the design variable, where $p>1$ and $q>0$ are penalty parameters for SIMP and RAMP, respectively.

Table 1 illustrates the computational results corresponding to the SIMP method, RAMP method, and Halpin Tsai, respectively.

It can be observed in Table 1 that the above amount of material is less at minimum computational period as compared to others. There are a number of conclusions according to above layouts which can be sketched as well as.

The SIMP method has lesser iteration numbers in comparison to the RAMP method. The results/outputs obtained by the RAMP method would have a smaller volume as compared to the SIMP method if the objective function has a minimum value. The results/outputs acquired through the RAMP method are superior than the SIMP method if the objective function is minimum volume. Moreover, the results of the SIMP method would be more accurate than the RAMP method if the objective function is minimum compliance. The iterations obtained through the RAMP method are more efficient than the SIMP method.

2.4. Super-Gaussian Projection. A super-Gaussian process is an illustrious machine learning tool for uncertainty quantification projects and is adopted in this article. It is assumed as piecewise linear profiles, and each linear profile contains four segments. Configuration of $i$ th profile and every $j$ th point which has the distance parameter are well-described; the super-Gaussian function is utilized for projection in topology optimization as follows:

$$
\phi_{i, j}=e^{-(1 / 2)\left(d_{i, j}^{2} / \gamma_{\phi}^{2}\right)^{\mu_{\phi}}}, \quad \text { for } j=1, \ldots, N_{\text {ele }},
$$

where $\mu_{\Phi}$ governs the curvature of the super-Gaussian projection. If two profiles are perpendicular or parallel concerning the desired position, each $i$ th point encloses by 

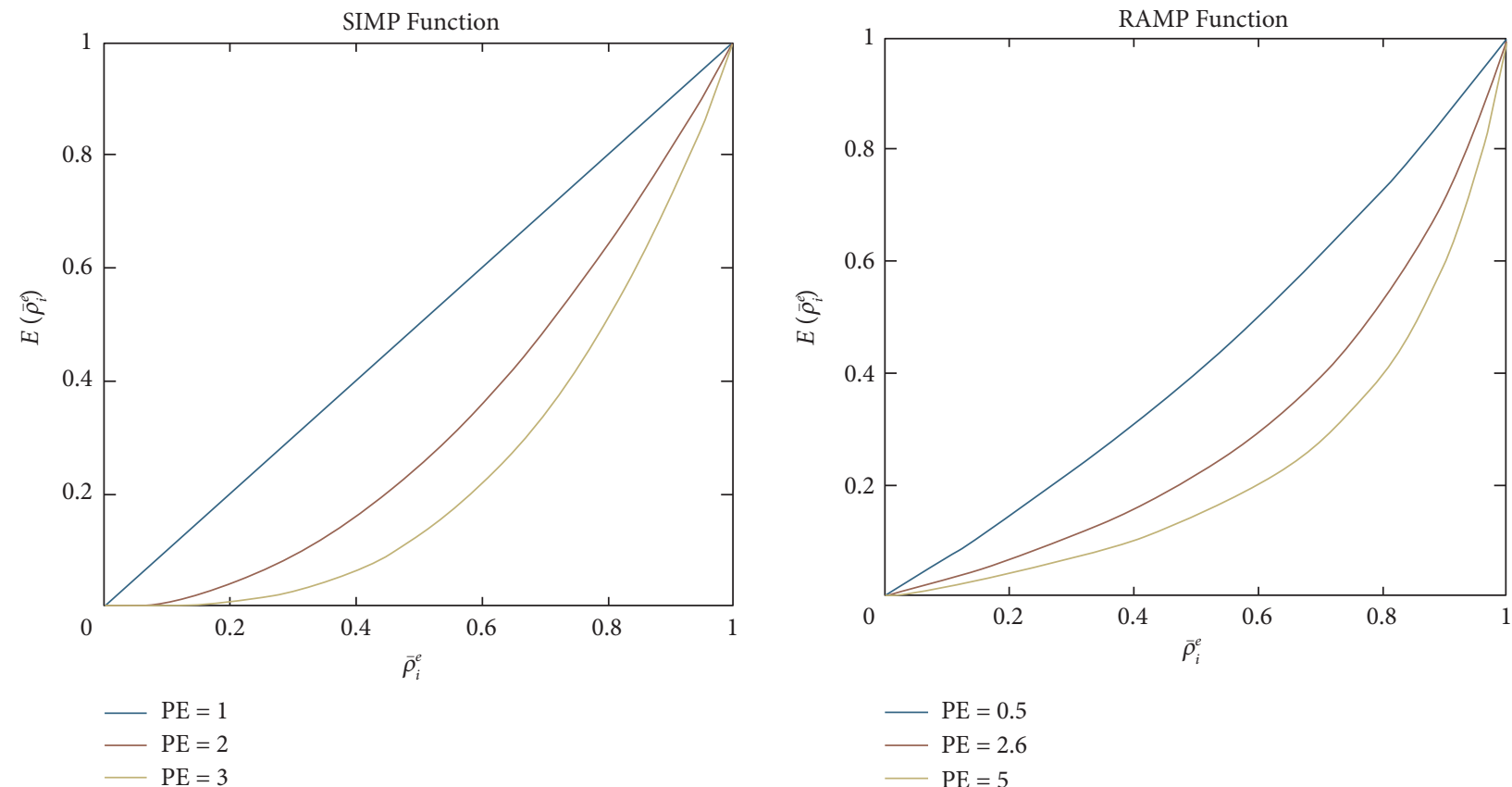

Figure 1: Experimental and numerical comparison layouts b/w the SIMP and RAMP models.

TABLE 1: Experimental and numerical comparison layouts b/w the SIMP and RAMP approaches.

\begin{tabular}{|c|c|c|c|}
\hline $240 * 120$ & SIMP & RAMP & Halpin Tsai \\
\hline \multicolumn{4}{|l|}{ Results } \\
\hline Total iterations & 400 & 400 & 400 \\
\hline Compliance & 88 & 87 & 83 \\
\hline $\mathrm{PE}$ & 3 & 0.9 & 1.5 \\
\hline
\end{tabular}

two distances $d_{i, 1}$ and $d_{i, 2}$ corresponding to two segments which are belonging to a profile.

The vicinity of the two profiles is correlated with the predicted variables $\Phi$, whose values are more significant than one if the two profiles are similar to each other. This scenario is an undesired activity that we want to discourage. We want to have a predicted vector $\Phi$ at most equal to one for points close to two segments with two different profiles:

$$
\begin{aligned}
& \phi_{i, j}=e^{-(1 / 2)\left(d_{i, j}^{2} / \gamma_{\phi}^{2}\right)^{\mu_{\phi}},} \\
& \text { for } j=1, \ldots, N_{\text {ele }}, \\
& \text { for all } i: \\
& \bar{d}_{i}^{2}=\min \left(\left[d_{1, i}^{2}, d_{2, i}^{2}\right]\right)=d_{\max }^{2}-\max \left(\left[d_{\max }^{2}-d_{1, i}^{2}, d_{\max }^{2}-d_{2, i}^{2}\right]\right) .
\end{aligned}
$$

The expression of equation (19) allows choosing merely minimum distance keen on according to the classification of maximum distance: $\phi_{i, j}=e^{-(1 / 2)\left(d_{i, j}^{2} / \gamma_{\phi}^{2}\right)^{\mu_{\phi}}}$,

for $j=1, \ldots, N_{\text {ele }}$,

for all $i$ :

$\bar{d}_{i}^{2}=d_{\max }^{2}-\left(\frac{\left(d_{\max }^{2}-d_{1, i}^{2}\right)^{q+1}+\left(d_{\max }^{2}-d_{2, i}^{2}\right)^{q+1}}{\left(d_{\max }^{2}-d_{1, i}^{2}\right)^{q}+\left(d_{\max }^{2}-d_{2, i}^{2}\right)^{q}}\right)$.

We utilize the distance filter $\tilde{d}_{i}^{2}$ to obtain an improved depiction of required layouts, concerning the suggested distance field $\bar{d}_{i}^{2}$, is given as

$$
\tilde{d}_{i}^{2}=\frac{\sum \sum_{j \in N_{i}} \omega\left(\Delta \mathbf{x}_{i j}\right) \bar{d}_{j}^{2}}{\sum_{j \in N_{e, i}} \omega\left(\Delta \mathbf{x}_{j}\right)} .
$$

Equation (20) can be rewritten in the matrix form as follows: 


$$
\tilde{d}^{2}=D\left(H_{s}^{\phi}\right)^{-1}\left(H^{\phi} \bar{d}_{j}^{2}\right)
$$

where entries $\left(H^{\Phi}\right)$ and $\left(H_{s}^{\Phi}\right)$ are vectors that are $\left[N_{\text {ele }} \times\right.$ $\left.N_{\text {ele }}\right]$ and $\left[N_{\text {ele }} \times 1\right]$. These vectors are described as

$$
\begin{aligned}
& H_{i j}^{\phi}=\omega\left(\Delta x_{i j}\right), \\
& H_{s, i}^{\phi}=\sum_{j} H_{i j} .
\end{aligned}
$$

In this paper, at the final stage, to project a parameterized smooth projection, the super-Gaussian function is as follows:

$$
\phi_{i}=e^{-(1 / 2)\left(\bar{d}_{i}^{2} / \gamma_{\phi}^{2}\right)^{\mu_{\phi}}}, \quad \text { for } j=1, \ldots, N_{\text {ele }} .
$$

\section{Control over Projected Entities}

This section expresses a critical, quite flexible topology optimization that can control many design features to mix projection and density-based design variables. It is assumed the volume constraint resting arranged the structure domain can be written as

$$
g_{0}(\rho)=\frac{\sum_{i=1}^{N_{\text {ele }}} \bar{\rho}_{i}^{d} v_{i}}{\sum_{i=1}^{N_{\text {ele }}} v_{i}}-g_{0, d}^{*} \leq 0
$$

where $v_{i}$ is representing the volume of the $i$ th finite element, whereas $g_{0 \text {,dil }}^{*}$ is the existing solid volume fraction inside the dilated design.

Control of the volume of material inside a particular region is significant during the manufacturing development of structural assemblies. It requires minimizing the volume of material for welded at the different subcomponent interfaces of the structural assembly. Such desired control may be obtained by utilizing the local volume constraints; it is useful merely for those underlying structures in the proposed projection region. In particular, we express the constraints mathematically as

$$
g_{1}(\rho, \mathbf{x})=\frac{\sum_{i=1}^{N_{\text {ele }}} \bar{\rho}_{i}^{d} v_{i} \phi_{i}(\mathbf{x})}{\sum_{i=1}^{N_{\text {ele }}} v_{i} \phi_{i}(\mathbf{x})}-g_{1, d}^{*} \leq 0,
$$

where $\Phi_{i}(x)$ is the projection function as a explicitly coordinate profile variable $x$ for configuration concerning the $i$ th element and $g_{0 \text {,dil }}^{*}$ is the existing solid volume fraction inside the dilated design in topology optimization. The above-written equation represents a volume constraint introduced merely on a subdomain classified through elements for which $\Phi=1$. Now, we present the density filter $\hat{\rho}_{i}$ components and calculate the average local material configuration of the neighboring $\widehat{N}_{i}$ values as

$$
\hat{\rho}_{i}=\frac{\sum_{j \in \widehat{N}_{i}} \bar{\rho}_{j}^{d}}{\sum_{j \in \widehat{N}_{i}}{ }^{1}} .
$$

Equation (27) can be rewritten in the matrix form as follows:

$$
\widehat{\rho}=D\left(H_{s}^{1 s}\right)^{-1}\left(H^{1 s} \bar{\rho}^{d}\right)
$$

where entries $\left(H^{1 s}\right)$ and $\left(H_{s}^{1 s}\right)$ are the matrix which are $\left[N_{\text {ele }} \times N_{\text {ele }}\right]$ and $\left[N_{\text {ele }} \times 1\right]$. These matrixes are described as

$$
\begin{aligned}
H_{i j}^{1 s}= & \omega_{i j}^{1 s}, \\
H_{s, i}^{1 s}= & \sum_{j} H_{i j}^{1 s}, \\
\omega_{i j}^{1 s}= & \begin{cases}1, & \text { if } j \in \widehat{N}_{i}, \\
0, & \text { otherwise, }\end{cases} \\
& \hat{\rho}_{i} \phi_{i} \leq \beta, \quad \text { for all } i=1, \ldots, N_{\text {ele }} .
\end{aligned}
$$

The abovementioned equation $N_{\text {ele }}$ constraints can be rested in terms of an average percentage of solid material inside a neighboring of every element on maximum Von Mises stress as follows:

$$
\max _{i}\left(\widehat{\rho}_{i} \phi_{i}\right) \leq \beta, \quad \text { for all } i=1, \ldots, N_{\text {ele }}
$$

The above maximum function is not appropriate for numerically optimized layout concerning first-order information because it cannot be differential. So, we adopt the Kreisselmeier-Steinhauser (KS) function of Von Mises or $p$ norm function [12] as follows:

$$
\left(\sum_{i=1}^{N_{\text {ele }}} \hat{\rho}_{i}^{p} \phi_{i}\right)^{(1 / p)} \leq\left(\sum_{i=1}^{N_{\text {ele }}} \beta^{p} \phi_{i}\right)^{(1 / p)}
$$

where $p$ is $p$ norm which is not similar as mentioned PE penalization in the modified solid isotropic material with penalization approach. However, the projected quantities and optimization evolution suppose nearby discrete $0-1$ values. After organizing these terms, we move next to the following constraints' computation:

$$
g_{2}(\hat{\rho}, \mathbf{x})=\left(\frac{\sum_{i=1}^{N_{\text {ele }}} \hat{\rho}_{i}^{p} \phi_{i}}{\sum_{i=1}^{N_{\text {ele }}} \phi_{i}}\right)^{(1 / p)}-\beta \leq 0 .
$$

Here, to introduce another constraint to standardize a projection profile lying on a piecewise segmented profile, assume a limitation is resting on the segmented profile. Limit on a maximum slope is calculated as

$$
\Delta x_{\max }=\frac{\Delta y}{\tan \left(\theta_{\max }\right)} .
$$

According to equation (30), the suggested constraint is mathematically calculated as

$$
g_{3}(\mathbf{x})=\max \left(\Delta x_{i}^{2}\right) \leq \Delta x_{\max }^{2}, \quad \text { for } i=1, \ldots, N_{\text {node }} .
$$

This obtained constraint seems like linear constraint for the shape variable $x_{i}$. This single constraint is helpful along with an optimization algorithm which is not adequate for the computation of linear control. Therefore, adopt a $p$ norm differentiable calculation as follows: 


$$
g_{3}(\mathbf{x})=\frac{1}{\Delta x_{\max }^{2}}\left(\sum_{i=1}^{N_{\text {node }}-1}\left(\Delta x_{i}^{2}\right)^{p}\right)^{(1 / p)}-1 \leq 0 .
$$

This resultant constraint is more effective to standardize to the optimized segmented projection profile. The proposed projection variables $\Phi_{i}$ allow to delegate distinct material characteristics to the elements rested inside the projection area. So, many can consign Young's modulus $E_{\Phi}$ to the element inside the projection region with the help of the modified solid isotropic material with penalization interpolation or RAMP technique as follows:

$$
\begin{aligned}
& E_{i}=E_{\min }+\left(E_{\max }-E_{\min } r_{E}-E_{\min }\right) \bar{\rho}_{i, e}^{\mathrm{PE}}, \\
& r_{E}=\frac{\left(E_{\max }-E_{\phi}\right)}{E_{\max }}, \\
& E_{i}=E_{\min }+\left(E_{\max }-E_{\min } r_{E}-E_{\min }\right)\left(\frac{\bar{\rho}_{i, e}}{\left(1+\operatorname{PE}\left(1-\bar{\rho}_{i, e}\right)\right)}\right), \\
& r_{E}=\frac{\left(E_{\max }-E_{\phi}\right)}{E_{\max }},
\end{aligned}
$$

where proposing $r_{E}$ parameter specify distinct Young's modulus to solid elements within the selected projection region, whereas the $\Phi_{i}$ projection variable must be equal to 1 . The predefined expression of equation (35) allows the delegate solid elements to the inner side beside the outer side of the projection region through utilizing Young's modulus $E_{\Phi}$ and $E_{\max }$. This scenario would be helpful during structural assembly computing of distinct welded interfaces. Amir and Lazarov [40] described a minimum length scale executed on black-and-white spaces is depicted along with every optimized layout by utilizing a circle whose diameter signifies the length scale. To control the dimensional irregularity of the length scale, use the density filter whose radius variation depends on the existing position of an element and the projection profile. The significance density filter representation can be as

$$
\tilde{\rho_{i}}=\frac{\sum_{j \in N_{i}} \omega\left(\Delta \mathbf{x}_{i j}, \phi_{i}\right) \rho_{j}}{\sum_{j \in N_{i}} \omega\left(\Delta \mathbf{x}_{i j}, \phi_{i}\right)},
$$

where this expression $\omega\left(\Phi x_{i j}, \Phi_{i}\right)$ defines the modified weight function:

$$
\omega\left(\Delta \mathbf{x}_{i j}, \phi_{i}\right)=\max \left(\bar{r}_{\min }\left(\phi_{i}\right)-\left\|\mathbf{x}_{j}-\mathbf{x}_{i}\right\|, 0\right) .
$$

In equation (38), the filter radius is described as

$$
\bar{r}_{\text {min }}\left(\phi_{i}\right)=r_{\text {min }}\left(1+\alpha \phi_{i}\right) \text {. }
$$

Here, $r_{\min }$ and $\alpha$ are the significance filter radius and filter radius amplification feature, respectively.

Equation (36) can be rewritten in the matrix form as follows:

$$
\widetilde{\rho}=D\left(H_{s}(\phi)\right)^{-1}(H(\phi) \rho),
$$

where entries $(H)$ and $\left(H_{s}\right)$ are vectors which are represented by $\left[N_{\text {ele }} \times N_{\text {ele }}\right]$ and $\left[N_{\text {ele }} \times 1\right]$. These vectors are described as

$$
\begin{aligned}
& H_{i j}=\omega\left(\Delta \mathbf{x}_{i j}, \phi_{i}\right), \\
& H_{s, i}=\sum_{j} H_{i j} .
\end{aligned}
$$

Here, the filter mentioned above the modified weight function interchange to another Gaussian weight proposed by [36]

$$
\omega_{G}\left(\Delta \mathbf{x}_{i j}, \phi_{i}\right)=\exp \left(-\left(\frac{\left\|\Delta \mathbf{x}_{j}-\mathbf{x}_{i}\right\|}{\bar{r}_{\min }\left(\phi_{i}\right) / 2}\right)^{n}\right) .
$$

The suggested Gaussian weight has the same layout, and the modified weight function resting $n$ must be equivalent to 2 , whereas the exp function and $n$ both are positive, respectively. With due respect to this significant development, we assume the aforementioned (27) can be rewritten as follows:

$$
\begin{aligned}
\hat{\rho}_{i} & =\frac{\sum_{j \in N_{i}} \omega\left(\Delta \mathbf{x}_{i j}\right) \bar{\rho}_{j}^{d}}{\sum_{j \in N_{i}} \omega\left(\Delta \mathbf{x}_{i j}\right)}, \\
\omega\left(\Delta \mathbf{x}_{i j}\right) & =\mathscr{H}\left(\max \left(\bar{r}_{\text {min }}\left(\phi_{i}\right)-\left\|\mathbf{x}_{j}-\mathbf{x}_{i}\right\|, 0\right)\right),
\end{aligned}
$$

where $\mathscr{H}$ represents the Heaviside function which gives 1 at nonzero input values of $x$; on the other side, it provides only 0 . This filter weight seems like the Gaussian weight function as the equation mentioned above. The weight $\omega_{G}$ further converges to homogenous through rising the nonzero values of $n$ and almost equivalent to 1 . Follow the improvement of the filter radius $r_{\min }$ resting upon the argument of exponent $n$ such that

$$
\begin{aligned}
\tilde{r}_{\text {min }} & =r_{\text {min }}(1+(1-2 / n)), \\
\widehat{r}_{\min }\left(\phi_{i}, n\right) & =\widetilde{r}_{\min }\left(1+\alpha \phi_{i}\right), \\
\omega_{G}\left(\Delta \mathbf{x}_{i j}, \phi_{i}\right) & =\exp \left(-\left(\frac{\left\|\mathbf{x}_{j}-\mathbf{x}_{i}\right\|}{\widetilde{r}_{\text {min }}\left(\phi_{i}\right) / 2}\right)^{n}\right) .
\end{aligned}
$$

Obtain the required length-scale control resting upon rising the nonzero values of $n$ due to $\widetilde{r}_{\text {min }}$. Pollini and Amir [33] introduced another constraint to govern the length scale along varying maximum feature size; the mathematical formulation of this constraint can be written down as

$$
\begin{aligned}
g_{4}(\widehat{\rho}, \mathbf{x}) & =\left(\frac{\sum_{i=1}^{N_{\text {el }}} \widehat{\rho}_{i}^{p}}{N_{\text {ele }}}\right)^{(1 / p)}-\beta \leq 0, \\
\hat{\rho}_{i} & =\frac{\sum_{j \in N_{i}} \omega_{G}\left(\Delta \mathbf{x}_{i j}, \widehat{r}_{\min }\left(\phi_{i}, n\right)\right) \bar{\rho}_{j}^{d}}{\sum_{j \in N_{i}} \omega_{G}\left(\Delta \mathbf{x}_{i j}, \widehat{r}_{\min }\left(\phi_{i}, n\right)\right)}, \quad \text { for all } i=1, \ldots, N_{\text {ele }} .
\end{aligned}
$$
$\{4,6\}$.
Here, exponent $n$ must be greater than to 2 , for example 


\section{Sensitivity Analysis}

The objective function variables are implicitly stated over the equilibrium equations in optimization problems. Utilizing the adjoint method, sensitivity analysis assumed to formulate the gradient of objective and constraint functions which rested upon the topological variables and segmented projection profile $x$ such as $g_{1}, g_{2}$, and $g_{4}$ although objective function $f$. Besides, the constraint $g_{3}$ merely rested upon the shape variable. The following gradient derivation of constraints $g_{1}, g_{2}, g_{3}$, and $g_{4}$ is already described in [37]. Utilizing the adjoint sensitivity method, to formulate the gradient of the objective can be found as

$$
\widehat{f}(\rho, \mathbf{x})=\mathbf{f}^{T} \mathbf{u}+\boldsymbol{\lambda}^{T}\left(\mathbf{K}\left(\rho_{e}, \mathbf{x}\right) \mathbf{u}-\mathbf{f}\right) .
$$

According to the equilibrium equation, if $\mathbf{K}\left(\rho_{e}, \mathbf{x}\right) \mathbf{u}-\mathbf{f}=0$, then $\mathbf{f}=\widehat{f}$. After satisfying the equilibrium condition, take the derivative of $\widehat{f}$ regarding the generic $\varsigma$ variable:

$$
\frac{\partial \widehat{f}}{\partial \varsigma}=\mathbf{f}^{T} \frac{\partial y}{\partial x}+\lambda^{T}\left(\frac{\partial \mathbf{K}}{\partial \varsigma} \mathbf{u}+\mathbf{K} \frac{\partial \mathbf{u}}{\partial \varsigma}\right)
$$

Removing the implicit derivative $(\partial u / \partial \varepsilon)$ in the equation mentioned above. Calculate the vector $\lambda$ to utilize the following equation:

$$
\mathbf{K}^{T} \boldsymbol{\lambda}=-\mathbf{f}
$$

Then,

$$
\frac{\partial f}{\partial \varsigma}=\lambda^{T} \frac{\partial \mathbf{K}}{\partial \varsigma} \mathbf{u}
$$

In our case, the objective function assumed is structural compliance. Then, the problem behaves like a self-adjoint. Consequently, we put $\lambda=-\mathbf{u}$ and to identify the derivative $(\partial \widehat{f} / \partial \varepsilon)$, regarding $\rho$, utilizing the chain rule:

$$
\begin{aligned}
\frac{\partial f}{\partial \rho_{i}} & =\sum_{j \in N_{i}}\left(\frac{\partial \hat{f}}{\partial \bar{\rho}_{j}^{e}} \frac{\partial \bar{\rho}_{j}^{e}}{\partial \tilde{\rho}_{j}}\right) \frac{\partial \tilde{\rho}_{j}}{\partial \rho_{i}}=\sum_{j \in N_{i}} H_{i j}\left(\frac{1}{H_{s, j}} \frac{\partial \hat{f}}{\partial \bar{\rho}_{j}^{e}} \frac{\partial \bar{\rho}_{j}^{e}}{\partial \tilde{\rho}_{j}}\right), \\
\therefore \frac{\partial \widehat{f}}{\partial \bar{\rho}_{i}^{e}} & =\lambda^{T} \frac{\partial \mathbf{K}}{\partial \bar{\rho}_{i}^{e}} \mathbf{u}, \\
\frac{\partial \mathbf{K}}{\partial \bar{\rho}_{i}^{e}} & =A_{i=1}^{N_{\text {ele }}} \operatorname{PE}\left(E_{\max }-E_{\min }\right)\left(\bar{\rho}_{i}^{e}\right)^{\mathrm{PE}-1} \mathbf{K}_{e, i}, \\
\text { or } \frac{\partial \mathbf{K}}{\partial \bar{\rho}_{i}^{e}} & =A_{i=1}^{N_{\text {ele }}}\left(E_{\max }-E_{\min }\right) \frac{(1+\mathrm{PE})}{\left(1+\mathrm{PE}\left(1-\bar{\rho}_{i}^{e}\right)\right)^{2} \mathbf{K}_{e, i}} .
\end{aligned}
$$

Here, $A$ is known as a matrix assembly operator, and the stiffness matrix also rests upon the shape coordinate of segmented profile and objective function $f$ variable:

$$
\begin{aligned}
\frac{\partial f}{\partial \rho_{i}} & =\sum_{k \in N_{j}}\left(\left(\frac{\partial \widehat{f}}{\partial \phi_{k}} \frac{\partial \phi_{k}}{\partial \widetilde{d}_{k}^{2}}\right) \frac{\partial \tilde{d}_{k}^{2}}{\partial \bar{d}_{j}^{2}}\right) \frac{\partial \bar{d}_{j}^{2}}{\partial \mathbf{x}_{i}} \\
& =\sum_{j \in N_{i}} H_{j k}^{\phi}\left(\frac{1}{H_{s, k}^{\phi}} \frac{\partial \hat{f}}{\partial \phi_{k}} \frac{\partial \phi_{k}}{\partial \tilde{d}_{k}^{2}}\right) \frac{\partial \bar{d}_{j}^{2}}{\partial \mathbf{x}_{i}}, \\
\therefore \frac{\partial \hat{f}}{\partial \phi_{i}} & =\lambda^{T} \frac{\partial \mathbf{K}}{\partial \phi_{i}} \mathbf{u}, \\
\frac{\partial \mathbf{K}}{\partial \phi_{k}} & =A_{i=1}^{N_{\text {ele }}} r_{E} E_{\max }\left(\bar{\rho}_{i}^{e}\right)^{\mathrm{PE}} \mathbf{K}_{e, i}, \\
\text { or } \frac{\partial \mathbf{K}}{\partial \phi_{k}} & =A_{i=1}^{N_{\text {ele }}} r_{E} E_{\max }\left(\frac{\bar{\rho}_{i}^{e}}{\left(1+\mathrm{PE}\left(1-\bar{\rho}_{i}^{e}\right)\right)}\right) \mathbf{K}_{e, i} .
\end{aligned}
$$

The calculation of the gradient of $g_{0}$ regarding $\rho$ is as

$$
\frac{\partial g_{o}}{\partial \rho_{i}}=\sum_{j \in N_{i}}\left(\left(\frac{\partial g_{o}}{\partial \bar{\rho}_{j}^{d}} \frac{\partial \bar{\rho}_{j}^{d}}{\partial \tilde{\rho}_{j}}\right) \frac{\partial \tilde{\rho}_{j}}{\partial \rho_{i}}\right)=\sum_{j \in N_{i}} H_{i j}\left(\frac{1}{H_{s, j}} \frac{\partial g_{o}}{\partial \bar{\rho}_{j}^{d}} \frac{\partial \bar{\rho}_{j}^{d}}{\partial \tilde{\rho}_{j}}\right) \text {. }
$$

To calculate the gradient of $g_{1}$ regarding $\rho$ similarly to the equation mentioned above, although constraint $g_{1}$ is belonging to function $x$, then

$$
\begin{aligned}
\frac{\partial g_{1}}{\partial \rho_{i}} & =\sum_{j \in N_{i}}\left(\left(\frac{\partial g_{o}}{\partial \bar{\rho}_{j}^{d}} \frac{\partial \bar{\rho}_{j}^{d}}{\partial \tilde{\rho}_{j}}\right) \frac{\partial \tilde{\rho}_{j}}{\partial \rho_{i}}\right)=\sum_{j \in N_{i}} H_{i j}\left(\frac{1}{H_{s, j}} \frac{\partial g_{1}}{\partial \bar{\rho}_{j}^{d}} \frac{\partial \bar{\rho}_{j}^{d}}{\partial \tilde{\rho}_{j}}\right), \\
\frac{\partial g_{1}}{\partial x_{i}} & =\sum_{k \in N_{j}}\left(\left(\frac{\partial g_{1}}{\partial \phi_{k}} \frac{\partial \phi_{k}}{\partial \tilde{d}_{k}^{2}}\right) \frac{\partial \tilde{d}_{k}^{2}}{\partial \bar{d}_{j}^{2}}\right) \frac{\partial \bar{d}_{j}^{2}}{\partial x_{i}} \\
& =\sum_{k \in N_{j}} H_{j k}^{\phi}\left(\frac{1}{H_{s, k}^{\phi}} \frac{\partial g_{1}}{\partial \phi_{k}} \frac{\partial \phi_{k}}{\partial \tilde{d}_{k}^{2}}\right) \frac{\partial \bar{d}_{j}^{2}}{\partial x_{i}}
\end{aligned}
$$

Now, calculate the gradient of $g_{2}$ regarding $\rho$ utilizing the chain rule as

$$
\begin{aligned}
\frac{\partial g_{2}}{\partial \rho_{i}} & =\sum_{j \in N_{i}}\left(\sum_{k \in N_{j}}\left(\frac{\partial g_{2}}{\partial \widehat{\rho}_{k}}\right) \frac{\partial \widehat{\rho}_{k}}{\partial \bar{\rho}_{j}^{d}} \frac{\partial \bar{\rho}_{j}^{d}}{\partial \widetilde{\rho}_{j}}\right) \frac{\partial \tilde{\rho}_{j}}{\partial \rho_{i}} \\
& =\sum_{j \in N_{i}} H_{i j}\left(\frac{1}{H_{s, j}} \sum_{k \in N_{j}} H_{j k}^{l s}\left(\frac{1}{H_{s, k}^{l s}} \frac{\partial g_{2}}{\partial \widehat{\rho}_{k}}\right) \frac{\partial \bar{\rho}_{j}^{d}}{\partial \widetilde{\rho}_{j}}\right) .
\end{aligned}
$$

Now, calculate the gradient of $g_{2}$ regarding the shape variables $x$ utilizing the chain rule as

$$
\begin{aligned}
\frac{\partial g_{2}}{\partial x_{i}} & =\sum_{k \in N_{j}}\left(\left(\frac{\partial g_{2}}{\partial \phi_{k}} \frac{\partial \phi_{k}}{\partial \tilde{d}_{k}^{2}}\right) \frac{\partial \tilde{d}_{k}^{2}}{\partial \bar{d}_{j}^{2}}\right) \frac{\partial \bar{d}_{j}^{2}}{\partial x_{i}} \\
& =\sum_{j \in N_{i}} H_{j k}^{\phi}\left(\frac{1}{H_{s, k}^{\phi}} \frac{\partial g_{2}}{\partial \phi_{k}} \frac{\partial \phi_{k}}{\partial \tilde{d}_{k}^{2}}\right) \frac{\partial \bar{d}_{j}^{2}}{\partial x_{i}} .
\end{aligned}
$$

To present the impact of filters with a radius variable keen on the sensitivity, gradient derivative of objective function regarding the coordinates of the projection $x_{i}$ can be found, respectively: 


$$
\begin{aligned}
\frac{\partial f}{\partial x_{i}} & =\frac{\partial g_{1}}{\partial x_{i}}+\sum_{k \in N_{j}} H_{j k}^{\phi}\left(\frac{1}{H_{s, k}^{\phi}} \frac{\partial \hat{f}}{\partial \bar{\rho}_{k}^{e}} \frac{\partial \tilde{\rho}_{k}^{e}}{\partial \tilde{\rho}_{k}} \frac{\partial \tilde{\rho}_{k}}{\partial \phi_{k}} \frac{\partial \phi_{k}}{\partial \tilde{d}_{k}^{2}}\right) \frac{\partial \bar{d}_{j}^{2}}{\partial x_{i}}, \\
\therefore \frac{\partial \tilde{\rho}_{i}}{\partial \phi_{i}} & =\frac{(\mathrm{d} \mathbf{H} \rho)_{i} / H_{s, i}-(\mathbf{H} \rho)_{i} \mathrm{~d} H_{s, i}}{H_{s, i}^{2}}, \\
\therefore \mathrm{d} H_{i j} & =\frac{\partial \omega_{G}\left(\Delta \mathbf{x}_{i j}, \phi_{i}\right)}{\partial \phi_{i}}, \\
\mathrm{~d} H_{s, i} & =\sum_{j} H_{i j} .
\end{aligned}
$$

In the end, taking the gradient derivative of $g_{4}$ regarding $\rho, x$ can be written as

$$
\begin{aligned}
\frac{\partial g_{4}}{\partial \rho_{i}}= & \sum_{j \in N_{i}}\left(\sum_{k \in N_{j}}\left(\frac{\partial g_{4}}{\partial \hat{\rho}_{k}}\right) \frac{\partial \widehat{\rho}_{k}}{\partial \bar{\rho}_{j}^{d}} \frac{\partial \bar{\rho}_{j}^{d}}{\partial \tilde{\rho}_{j}}\right) \frac{\partial \tilde{\rho}_{j}}{\partial \rho_{i}} \\
= & \sum_{j \in N_{i}} H_{i j}\left(\frac{1}{H_{s, j}} \sum_{k \in N_{j}} H_{j k}^{l s}\left(\frac{1}{H_{s, k}^{l s}} \frac{\partial g_{4}}{\partial \hat{\rho}_{k}}\right) \frac{\partial \bar{\rho}_{j}^{d}}{\partial \tilde{\rho}_{j}}\right), \\
\frac{\partial g_{4}}{\partial x_{i}}= & \sum_{k \in N_{j}} H_{j k}^{\phi}\left(\frac{1}{H_{s, k}^{\phi}} \frac{\partial g_{4}}{\partial \phi_{k}} \frac{\partial \phi_{k}}{\partial \tilde{d}_{k}^{2}}\right) \frac{\partial \bar{d}_{j}^{2}}{\partial x_{i}} \\
& +\sum_{k \in N_{j}} H_{j k}^{\phi}\left(\frac{1}{H_{s, k}^{\phi}} \frac{\partial g_{4}}{\partial \bar{\rho}_{k}^{e}} \frac{\partial \bar{\rho}_{k}^{e}}{\partial \tilde{\rho}_{k}} \frac{\partial \tilde{\rho}_{k}}{\partial \phi_{k}} \frac{\partial \phi_{k}}{\partial \tilde{d}_{k}^{2}}\right) \frac{\partial \bar{d}_{j}^{2}}{\partial x_{i}} .
\end{aligned}
$$

\section{Numerical Discussion}

Two-dimensional numerical examples demonstrate the proficiency and efficiency of the recommended modified mixed projection density-based optimization method. As assumed, moving limits in topology optimization for the geometric variable, which identifies the shape of the projection profile besides density variables, are equivalent to 0.005 and 0.2 , respectively. In topology optimization, the MBB beam is a famous numerical assessment and point of the reference problem. Parameter $\gamma$ governs the sharpness of projected smooth projection of design variable $\widetilde{\rho}$ within the eroded $\bar{\rho}^{e}$, intermediate $\bar{\rho}^{i}$, and dilated $\bar{\rho}^{d}$ outcomes. Furthermore, Young's modulus $E_{\max }$ is equivalent to 1 for distribution material, $E_{\min }$ is considered $1 \times 10^{-6}$ a small stiffness is allotted to void spaces for the stiffness matrix not to be becoming singular, and the Poisson ratio is equivalent to $v=0.3$, respectively.

There are many descriptive numerical cases to show the well-intentioned significance of the proposed modified method. Here, we present the initial structure domain of the MBB beam in Figure 2, whereas the participating material characteristic, the applied external loads, and geometry data, respectively. Following case (a), left side is clamped and the other opposite side force is applied at the bottom. In case (b), the left-hand edge is completely fastened, and a force $f$ is vertically operated on the righthand of the middle side. Utilize the method of moving asymptotes as an optimizer to sort out the optimization problem [41].

Example 1. In the first example, the optimized cantilever structure problem under examination is presented in Figure 3. We endow with a moderately good evaluation of the suggested modified and merge projection density-based optimization algorithm to treat along with numerous constraints. For the finite element structure domain dimensions, nelx and nely are $3 \times 1$ where elements are placed horizontally and vertically, respectively. The primary purpose of the representation of black and blue dashed lines is to differentiate between the chosen outer boundaries and the actual structure of the design domain. $\mathrm{PE}=3$ is used as a solid isotropic material penalization constant factor and substituted for $r E=0.5$ which is half of Young's modulus $E_{\max }$. Volume constraint is 40 percent, density filter is $r$, generally, $r_{\min }=10$ is so-called density filter radius utilized to obtain minimized length scale, $r_{\max }$ is so-called density filter radius used to obtain maximized length scale, in projection profile, $r_{\Phi}=10$ is filter distance field, and $g_{0}^{*}$ is the suitable volume fraction for $g_{0}$ constraint. We follow the focus obtained in an impressive result of the MBB beam. Moreover, in the projection profile, there are some 6 nodes that are fixed vertically at $y$ coordinates and moveable $x$-coordinates besides 5 segments.

After modifying this suggested approach, we get hold of optimized stable convergence as a reference result, respectively.

Example 2. This example demonstrates the opportunity to optimize a structure with an interface between substructures also concurrently optimized. The existence of welding materials and various mechanical characteristics joining the different substructures are considered. The variable shape of the project file is used to represent the connection among the substructures. The finite element structure domain dimensions nelx and nely are $3 \times 1$. There are two volume constraints to the total volume constraint $g_{0}$ and $g_{1}$; the constraint is a local volume that is operated at the projection region, respectively. To acquire supplementary efficient shapes' layout of optimized projection profile, we utilized $g_{3}$ as a third constraint. The projection profile organized with physical representation of a node in the following layout figure is a red circle, where the horizontally coordinate $x$ belongs to a geometric variable. Geometric variables $x_{i}$, where $i=1, \ldots, 6$, are firstly synchronized as 0.5 , and their bounds which represent the figure are a magenta-colored dash line. Assume moving limits in topology optimization for the geometric variable, which identifies the shape of the projection profile besides density variables equivalent to 0.005 and 0.2 .

It can be observed in Figure 4(a) that the above amount of material is less at minimum computational period as compared to the reference optimized result and at the exact value of volume fraction, respectively. We initially rested on 


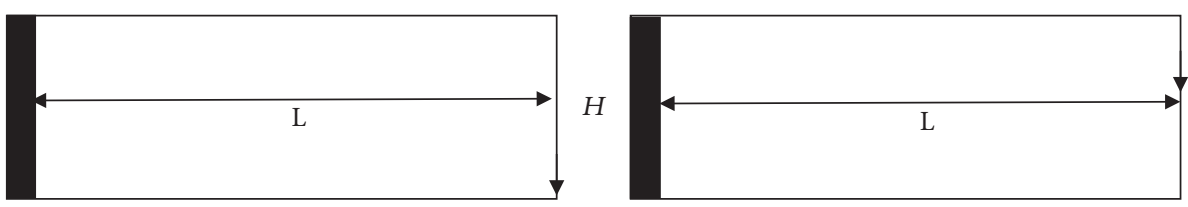

Figure 2: The initial structure domain of the MBB beam.

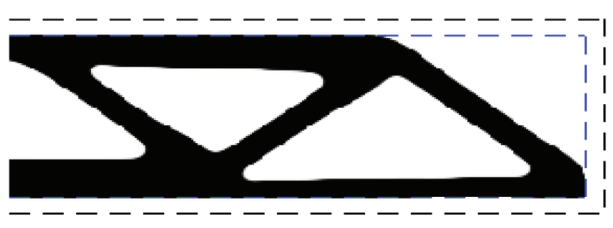

(a)

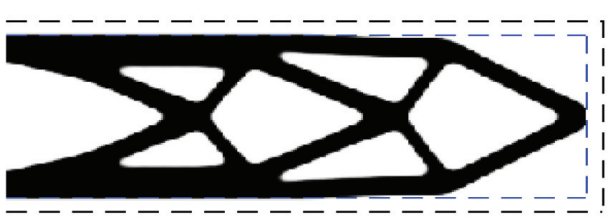

(b)

Figure 3: Beam FE structure domain dimension $3 \times 1$. (a) Final compliance $f=194$, iterations $=550$. (b) Final compliance $f=187$, iterations $=400$.

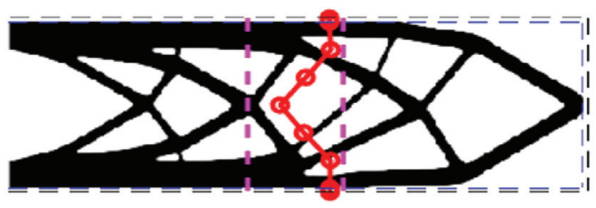

(a)

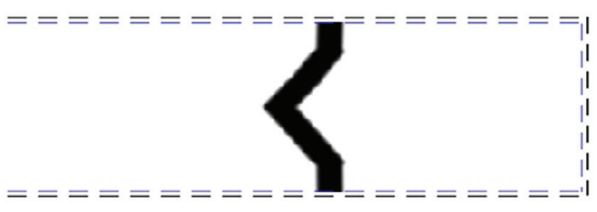

(b)

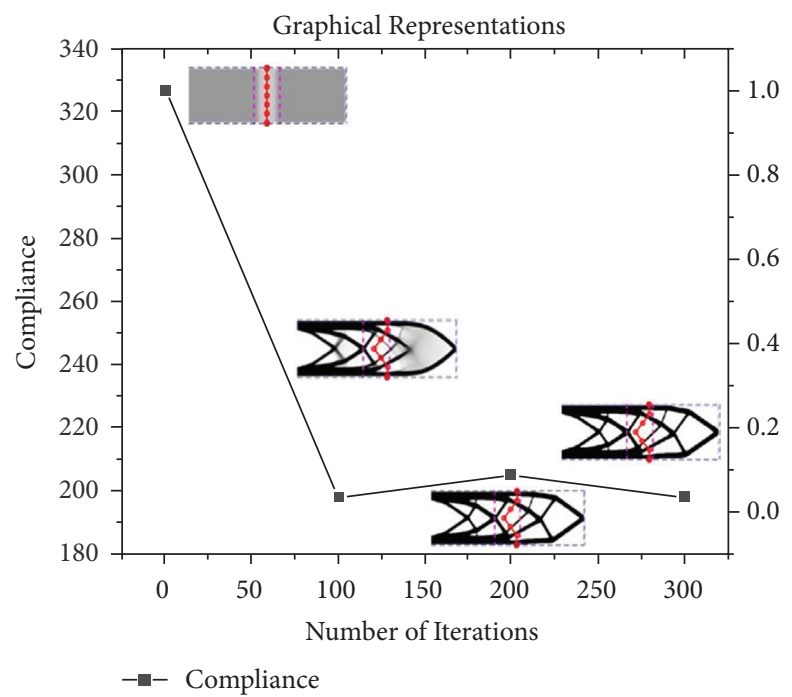

(c)

FIGURE 4: Optimized topologies and compliance for various constraints values. (a) $f=194, r_{\min }=3, r_{\phi}=3, r_{\max }=5, \gamma_{\Phi}=20$, and iterations $=300$. (b) Shape of optimized projection area. (c) Graphical representation.

the value $\mu_{\Phi}=1.25$ along increment of 1.4142 as well as in Figure 5; also, we take $\mu_{\Phi}=1.50$ along with the increment of 1.4142 at every sequel setup, which stops at a maximum value of 5 . Here, $\mu_{\Phi}$ governs the curvature of the superGaussian utilized for the projection. Here, case (b) shows the final shape of the optimized projection area and case (c) is a graphical representation between the final compliance result and the total number of iterations along with obtained optimized history.
Example 3. Here, we represent 2D numerically example to make evident the proficiency and efficiency of the recommended algorithm. We optimize the MBB beam utilizing the modified solid isotropic material with penalization; for the finite element structure domain dimensions, nelx and nely are $300 \times 100$, where elements are placed horizontally and vertically, respectively. There are two volume constraints to the total volume constraints $g_{0}$ and $g_{1}$; the constraint is a local volume that is operated at the projection region, respectively. 


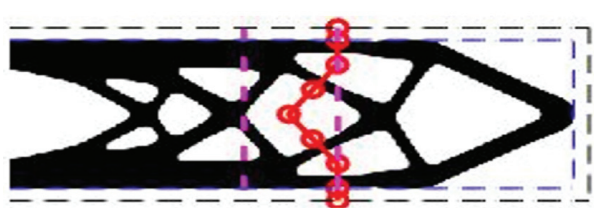

(a)

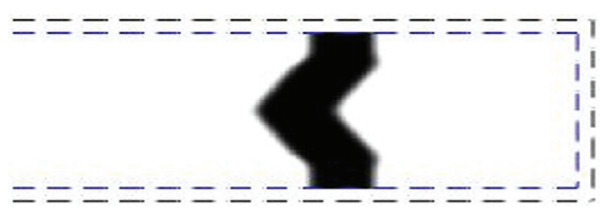

(b)

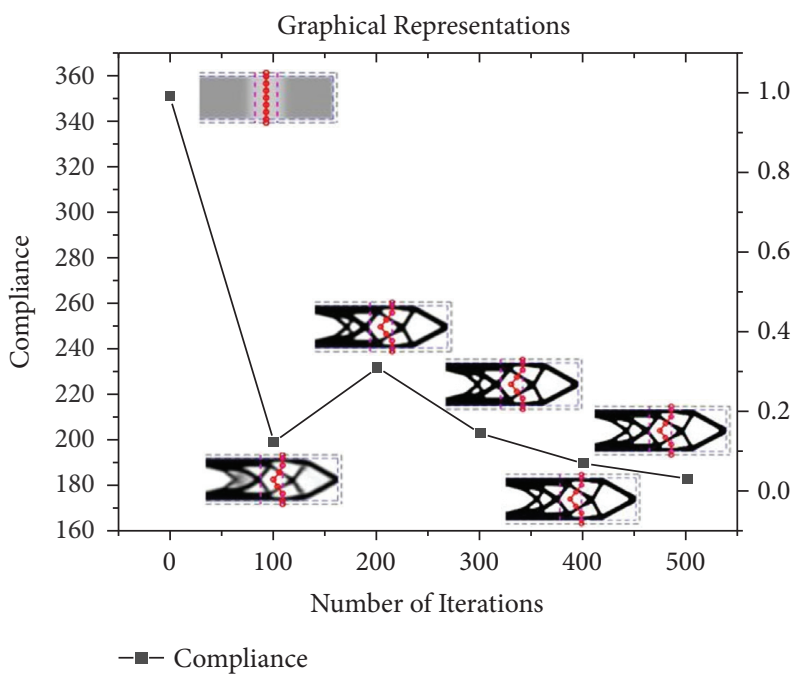

(c)

FIgURE 5: Optimized topologies and compliance for various constraints values. (a) $f=178, r_{\min }=10, r_{\phi}=10, r_{\max }=10, \gamma_{\Phi}=32$, and iterations $=50$. (b) Shape of optimized projection area. (c) Graphical representation.

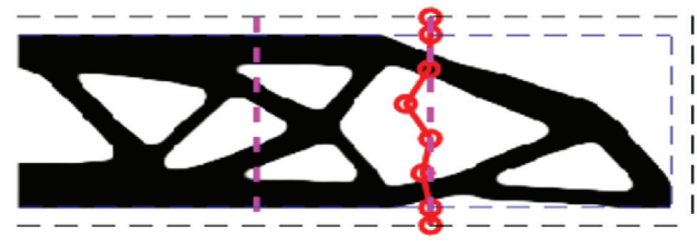

(A) $f=194, r_{\text {min }}=10, r_{\phi}=10, r_{\text {max }}=10, \gamma_{\phi}=20$, Iter $=550$

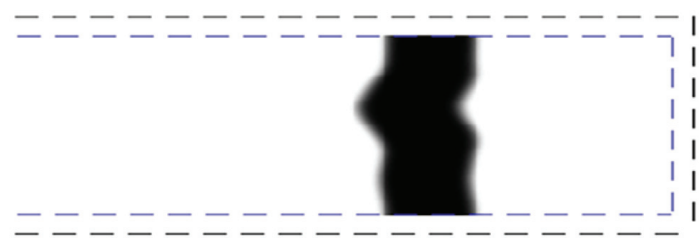

(B) Shape of Optimized Projection area

(a)

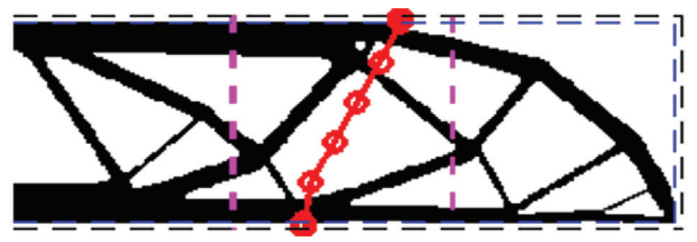

(A) $f=229, r_{\text {min }}=3, r_{\phi}=3, r_{\text {max }}=5, \gamma_{\phi}=32$, Iter $=350$

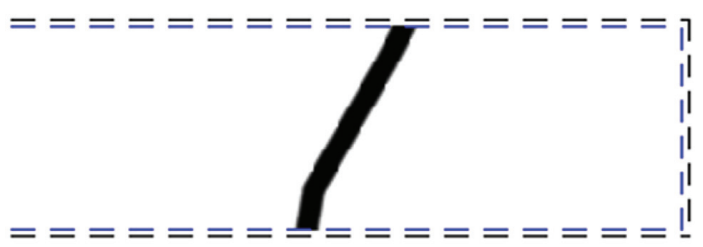

(B) Shape of Optimized Projection area

(b)

Figure 6: Optimized topologies and compliance for various constraints values. (a) $x_{\mathrm{lb}}=.40, x_{\mathrm{ub}}=0.60$. (A) $f=194, r_{\min }=10, r_{\phi}=10$, $r_{\max }=10, \gamma_{\Phi}=20$, and iter $=550$. (B) Shape of the optimized projection area. (b) $x_{\mathrm{lb}}=.25, x_{\mathrm{ub}}=0.75$. (A) $f=229, r_{\min }=3, r_{\phi}=3, r_{\max }=5$, $\gamma_{\Phi}=32$, and iter $=350$. (B) Shape of the optimized projection area.

To acquire supplementary efficient shapes' layout of optimized projection profile, we utilized $g_{3}$ as a third constraint. The projection profile organized with physical representation of a node in the following layout figure is a red circle, where the horizontally coordinate $x$ belongs to a geometric variable. Geometric variables $x_{i}$, where $i=1, \ldots, 6$, are firstly synchronized as 0.5 , and their bounds, which are represented in figure as a magenta-colored dash line. Parameters such as density filter radius is $r_{\min }$; generally, $r_{\max }$ is for maximum length scale control, $r_{\phi}$ filter is utilized for transition corners in segment cut, and $\gamma_{\Phi}$ is helped to define the specified region, where $g_{1}$ constraint is functional. The threshold projection parameters of the eroded intermediate are dilated, where $\eta_{e}=$ 0.52 and $\eta_{d}=0.48$, and $\gamma$ governs the sharpness of the 
projected smooth projection. In the optimization procedure, the intended dilated field vector $\bar{\rho}^{d}$ is utilized for evaluating the volume constraint, whereas the eroded $\bar{\rho}^{e}$ density field vector utilizes the calculation of the compliance and structural stiffness.

The above focused numerical topology optimized example unique layouts which are depicted in Figure 6 at minimum computational period.

The fundamental goal is to find an optimized topology by simulation. This topology imposes volume limitation, which is revised locally in the projection area. This condition may interpret the requirement for minimizing the dimension of the structural elements at the interface of the various parts created distinctly and later bring together. With the help of the proposed technique, this impressive remarkable optimized layout utilizing the minimized amount of material in a subportion of the design domain is gained. This obtained optimal result is more flexible than the optimized reference result, although both have approximately the same volume fraction. Despite that, in this focus example, we utilized the total amount of material as smaller than the reference layout at the interface area.

\section{Conclusion}

This article proposed a novel methodology that is a combination of two well-known implicit projections and modified explicit density-based formulation techniques and computational procedure. The said procedure is used to drop down the dimensions of structural elements. This dimension reduction is at the interface of the various parts that are manufactured distinctly and later assembled. These two representations coupled with explicit geometric entities that make availability of the specific control are provided, and the assigned domain and modified implicit entity make availability of structure freedom. However, geometric representations are linearly piecewise segments. There are numerous benefits of the combination of projection, and density-based optimized formulation methodology can be summarized. First, due to coupling, the shape and topological variables are helpful for prestressed concrete optimization. This existing work inspires by the optimal strategy of 2-dimensional structural assemblies.

The applicability of the suggested technique illustrates numerous numerical example tests. Section 5 obtained layouts which magnificently indicate such selective limitations in the assigned domain and provide the complete freedom of modified density-based factors elsewhere.

\section{Data Availability}

Data and supplementary materials are available upon request and are not available in public.

\section{Conflicts of Interest}

The authors declare that they have no conflicts of interest to report with respect to the present study.

\section{Acknowledgments}

The authors are thankful to Professor Krister Svanberg for providing the program code of MMA. The authors wish to acknowledge the financial support from the National Natural Science Foundation of Beijing (2182067) and the Fundamental Research Funds of the Central Universities (2018ZD09).

\section{Supplementary Materials}

The MATLAB source codes for all examples are provided as per your contact to the authors. (Supplementary Materials)

\section{References}

[1] M. P. Bendsøe and N. Kikuchi, "Generating optimal topologies IN structural design using a homogenization method," Computer Methods in Applied Mechanics and Engineering, vol. 71, 1988.

[2] T. H. Nguyen, G. H. Paulino, J. Song, and C. H. Le, "A computational paradigm for multiresolution topology optimization (MTOP)," Structural and Multidisciplinary Optimization, vol. 41, no. 4, pp. 525-539, 2010.

[3] E. T. Filipov, J. Chun, G. H. Paulino, and J. Song, "Polygonal multiresolution topology optimization (PolyMTOP) for structural dynamics," Structural and Multidisciplinary Optimization, vol. 53, no. 4, pp. 673-694, 2016.

[4] Q. X. Lieu and J. Lee, "Multiresolution topology optimization using isogeometric analysis," International Journal for $\mathrm{Nu}$ merical Methods in Engineering, vol. 112, no. 13, pp. 20252047, 2017.

[5] J. Wu, N. Aage, R. Westermann, and O. Sigmund, "Infill optimization for additive manufacturing-approaching bonelike porous structures," IEEE Transactions on Visualization and Computer Graphics, vol. 24, no. 2, pp. 1127-1140, 2017.

[6] K. Long, C. Gu, X. Wang et al., "A novel minimum weight formulation of topology optimization implemented with reanalysis approach," International Journal for Numerical Methods in Engineering, vol. 120, no. 5, pp. 567-579, 2019.

[7] J. A. Sethian, "A fast marching level set method for monotonically advancing fronts," Proceedings of the National Academy of Sciences, vol. 93, no. 4, pp. 1591-1595, 1996.

[8] N. Saeed, K. Long, and A. Rehman, "A review of structural optimization techniques for wind turbines," in Proceedings of the $20203 r$ International Conference on Computing, Mathematics and Engineering Technologies (iCoMET), pp. 18, Sukkur, Pakistan, January 2020.

[9] O. M. Querin, G. P. Steven, and Y. M. Xie, "Evolutionary structural optimisation (ESO) using a bidirectional algorithm," Engineering Computations, vol. 15, no. 8, pp. 1031-1048, 1998.

[10] J. Liu, G. Wen, and Y. M. Xie, "Layout optimization of continuum structures considering the probabilistic and fuzzy directional uncertainty of applied loads based on the cloud model," Structural and Multidisciplinary Optimization, vol. 53, no. 1, pp. 81-100, 2016.

[11] Y. Zhang, M. Xiao, H. Li, and L. Gao, "Topology optimization of material microstructures using energy-based homogenization method under specified initial material layout," Journal of Mechanical Science and Technology, vol. 33, no. 2, pp. 677-693, 2019. 
[12] V. Keshavarzzadeh, R. M. Kirby, and A. Narayan, "Stressbased topology optimization under uncertainty via simulation-based Gaussian process," Computer Methods in Applied Mechanics and Engineering, vol. 365, Article ID 112992, 2020.

[13] O. Amir and E. Shakour, "Simultaneous shape and topology optimization of prestressed concrete beams," Structural and Multidisciplinary Optimization, vol. 57, no. 5, pp. 1831-1843, 2018.

[14] X. Wang, K. Long, V.-N. Hoang, and P. Hu, “An explicit optimization model for integrated layout design of planar multi-component systems using moving morphable bars," Computer Methods in Applied Mechanics and Engineering, vol. 342, pp. 46-70, 2018.

[15] K. Long, X. Wang, and X. Gu, "Multi-material topology optimization for the transient heat conduction problem using a sequential quadratic programming algorithm," Engineering Optimization, vol. 50, no. 12, pp. 2091-2107, 2018.

[16] J. Petersson and O. Sigmund, "Slope constrained topology optimization," International Journal for Numerical Methods in Engineering, vol. 41, no. 8, pp. 1417-1434, 1998.

[17] T. A. Poulsen, "A new scheme for imposing a minimum length scale in topology optimization," International Journal for Numerical Methods in Engineering, vol. 57, no. 6, pp. 741-760, 2003.

[18] J. K. Guest, J. H. Prévost, and T. Belytschko, "Achieving minimum length scale in topology optimization using nodal design variables and projection functions," International Journal for Numerical Methods in Engineering, vol. 61, no. 2, pp. 238-254, 2004.

[19] J. K. Guest, A. Asadpoure, and S.-H. Ha, "Eliminating betacontinuation from heaviside projection and density filter algorithms," Structural and Multidisciplinary Optimization, vol. 44, no. 4, pp. 443-453, 2011.

[20] J. K. Guest, "Imposing maximum length scale in topology optimization," Structural and Multidisciplinary Optimization, vol. 37, no. 5, pp. 463-473, 2009.

[21] J. K. Guest, "Topology optimization with multiple phase projection," Computer Methods in Applied Mechanics and Engineering, vol. 199, no. 1-4, pp. 123-135, 2009.

[22] J. K. Guest, "Optimizing the layout of discrete objects in structures and materials: a projection-based topology optimization approach," Computer Methods in Applied Mechanics and Engineering, vol. 283, pp. 330-351, 2015.

[23] S. Chen, M. Y. Wang, and A. Q. Liu, "Shape feature control in structural topology optimization," Computer-Aided Design, vol. 40, no. 9, pp. 951-962, 2008.

[24] J. Luo, Z. Luo, S. Chen, L. Tong, and M. Y. Wang, "A new level set method for systematic design of hinge-free compliant mechanisms," Computer Methods in Applied Mechanics and Engineering, vol. 198, no. 2, pp. 318-331, 2008.

[25] W. Zhang, W. Zhong, and X. Guo, "An explicit length scale control approach in SIMP-based topology optimization," Computer Methods in Applied Mechanics and Engineering, vol. 282, pp. 71-86, 2014.

[26] G. Michailidis, Manufacturing constraints and multi-phase shape and topology optimization via a level-set method, 2014.

[27] Q. Xia and T. Shi, "Constraints of distance from boundary to skeleton: for the control of length scale in level set based structural topology optimization," Computer Methods in Applied Mechanics and Engineering, vol. 295, pp. 525-542, 2015.

[28] J. A. Norato, B. K. Bell, and D. A. Tortorelli, “A geometry projection method for continuum-based topology optimization with discrete elements," Computer Methods in Applied Mechanics and Engineering, vol. 293, pp. 306-327, 2015.

[29] Z. Meng and B. Keshtegar, "Adaptive conjugate single-loop method for efficient reliability-based design and topology optimization," Computer Methods in Applied Mechanics and Engineering, vol. 344, pp. 95-119, 2019.

[30] W. Zhang, J. Chen, X. Zhu et al., "Explicit three dimensional topology optimization via Moving Morphable Void (MMV) approach," Computer Methods in Applied Mechanics and Engineering, vol. 322, pp. 590-614, 2017.

[31] S. Watts and D. A. Tortorelli, "A geometric projection method for designing three-dimensional open lattices with inverse homogenization," International Journal for Numerical Methods in Engineering, vol. 112, no. 11, pp. 1564-1588, 2017.

[32] Z. Meng, Y. Pang, Y. Pu, and X. Wang, "New hybrid reliability-based topology optimization method combining fuzzy and probabilistic models for handling epistemic and aleatory uncertainties," Computer Methods in Applied Mechanics and Engineering, vol. 363, Article ID 112886, 2020.

[33] N. Pollini and O. Amir, "Mixed projection-and density-based topology optimization with applications to structural assemblies," Structural and Multidisciplinary Optimization, vol. 61, no. 2, pp. 687-710, 2019.

[34] T. E. Bruns and D. A. Tortorelli, "Topology optimization of non-linear elastic structures and compliant mechanisms," Computer Methods in Applied Mechanics and Engineering, vol. 190, no. 26-27, pp. 3443-3459, 2001.

[35] S. Xu, Y. Cai, and G. Cheng, "Volume preserving nonlinear density filter based on heaviside functions," Structural and Multidisciplinary Optimization, vol. 41, no. 4, pp. 495-505, 2010.

[36] O. Sigmund, "Morphology-based black and white filters for topology optimization," Structural and Multidisciplinary Optimization, vol. 33, no. 4-5, pp. 401-424, 2007.

[37] F. Wang, B. S. Lazarov, and O. Sigmund, "On projection methods, convergence and robust formulations in topology optimization," Structural and Multidisciplinary Optimization, vol. 43, no. 6, pp. 767-784, 2011.

[38] O. Sigmund and S. Torquato, "Design of materials with extreme thermal expansion using a three-phase topology optimization method," Journal of the Mechanics and Physics of Solids, vol. 45, no. 6, pp. 1037-1067, 1997.

[39] M. Stolpe and K. Svanberg, "An alternative interpolation scheme for minimum compliance topology optimization," Structural and Multidisciplinary Optimization, vol. 22, no. 2, pp. 116-124, 2001.

[40] O. Amir and B. S. Lazarov, "Achieving stress-constrained topological design via length scale control," Structural and Multidisciplinary Optimization, vol. 58, no. 5, pp. 2053-2071, 2018.

[41] K. Svanberg, "The method OF moving asymptotes-a new method for structural optimization," Numerical Methods in Engineering, vol. 24, pp. 359-373, 1987. 